\title{
Barriers of Discussion Concerning Sexual and Reproductive Health Issues Among Adolescents and Parents, Hawassa, SNNPR, Ethiopia
}

\author{
Sailaja Busi* and Nana Chea \\ Department of Nursing \& Midwifery, Hawassa University, Ethiopia
}

Received: December 02, 2017; Published: December 14, 2017

*Corresponding author: Sailaja Busi, Department of Nursing \& Midwifery, Hawassa University, College of Medicine \& Health Sciences, Ethiopia; Email: sailajabusi@gmail.com

Abstract

Background: Adolescence is a period of high developmental changes in physical, mental and social conditions. In this period the adolescent youth fails to control their emotions, listen to parents' advice and begins to indulge them in unhealthy behavioral activities. In general, the age group from 10 to 19 years is classified as adolescent. Many adolescents often lack strong and stable relationships with their parents to openly discuss about reproductive health concerns. Similarly, about $55.7 \%$ of the secondary and preparatory school students were not discussed on SRH issues.

Objective: To assess the barriers of discussion among adolescents concerning sexual and reproductive health issues among Preparatory Schools students of Hawassa City, SNNPR, Ethiopia.

Methods: - Descriptive cross sectional survey design was conducted among Addis Ketema and Tabor preparatory schools students in Hawassa City, from December to June 2016. Then a multistage sampling technique was employed to select 344 eligible study participants, the data were collected by using closed ended questionnaire and analyzed.

Result: - The majority (61\%) of respondents did not discused sexual matters with their parents. The results of binary logistic regression revealed that, the adolescents who follow social media $(B=1.497, p<0.05)$, use condom $(B=2.296, p<0.05)$, father education $(B=2.398, p<0.05)$ and mother education $(B=1.582, p<0.05)$ have positive and significant effect on discussion between the parents and adolescents on SRH issues. Culture of adolescents and parents regarding sex related issues whereas, ever had experienced sexual pleasure $(B=-1.466, p<0.01)$, amount of money the adolescent got monthly $(B=-2.127, p<0.01)$, talk to friend about sex related issues $(B=-2.361, p<0.01)$, and feel shame to talk to family about sex related issues $(B=-0.868, p<0.05)$ have negative and significant effect on discussion between the adolescents and parents regarding sex related issues.

Conclusion: The major factors that hindered parent-adolescent discussion were ever had experienced sexual pleasure, follow social media, use condom, parent education, amount of money given to adolescents, talk to friends about sex related issue, and feel shame. Furthermore, parents had limited access to sexuality information which could perpetuate beliefs, taboos and negative attitudes that hindered them from discussing sexual matters with adolescents.

Keywords: Adolescent; Barriers; Discussion

\section{Introduction}

Adolescence is a period of high developmental changes in physical, mental and social conditions. In this period the adolescents fails to control their emotions, listen to parents' advice and begins to indulge them in unhealthy behavioral activities. The youth is categorized into three age-groups 10 to 14 years old as teenage, 15 to 19 year as early adolescent and 20 to 24 years as post-adolescent. In general, the age group from 10 to 19 years is classified as adolescent [1]. The problems of adolescents arise from lack of understanding and proper response to the changes that occur during development, due to emotional behavior, peer pressure and lack of experience of prevailing social system and its interactions. Therefore, adolescents should learn about human reproductive system; reproductive organs and their functions, problems related to adolescence and in particular about an appropriate family responsibility. This undertaking should prepare the adolescent to feel responsible; to aspire and/or anticipate for a better future; to overcome problems and hurdles of life and eventually emerge equipped with the necessary knowledge and skills and physically strong and fit. The adolescent should be able to differentiate useful 
and harmful behavior and practices and feel responsible to protect others from danger.

In this respect, families and communities have important roles to play. In order for the adolescent to become effective head of the family, the adolescent should know about the definition of the family and its historical development and relationships [2]. The prevailing potential sources of SRH information for the young people are their peers whom their knowledge are infirmed/equally ignorant or from school which is blamed for the lack of sustainable behavioral changes or from media and religious institutions that occur infrequently [3]. Research showed that, the conservative norm and taboos on sexuality, and ill-preparation have largely limited the parents' involvement on SRH discussion with their children. However, remarkably, because of the devastating HIV/ AIDS problems, parental engagement has recently been receiving more attention [4]. A review on the magnitude of parent-adolescent discussion about HIV/AIDS in Sub-Saharan Africa reported was 8\%-80\% 5. Adolescents who communicate SRH matters with their parents are less likely to engage in unsafe sex [6]. The discussion is associated with adolescents' age, parental education, and parent types [7]. Parents and adolescents have more communicated on abstinence, pregnancy and HIV/AIDS while they communicated rarely on condom and other contraceptives [8].

About two-thirds of the Ethiopia's populations are young and they are the ones whose reproductive health services utilization is low and are the perpetrated with various sexual and reproductive health problems [9]. Adolescent discussion with parents regarding sexual and reproductive health issues protects the young from engaging in risky sexual practices and associated adverse health consequences [10]. Parents often have the power to guide children's development in sexual health matters, encouraging them to practice reasonable sexual behavior and develop good personal decision making skills [11]. Increased parent-child discussion leads to awareness and reduction in risk taking behaviors [12]. When young people feel unconnected to home and family, they may become involved in activities that put their health and wellbeing at risk [13]. However, many adolescents often lack strong and stable relationships with their parents or other adults who are necessary, to openly discuss reproductive health concerns. Therefore, many teenagers do not have access to reliable information regarding their reproductive health needs [14]. Africa accounted about four-fifths of the estimated five millions of young people living with HIV [15] and the others have been inflicting among one fourth of the four millions of adolescents unsafe abortion was observed [1].

The 2011 Ethiopia Demographic and Health Survey (EDHS) report indicated that, $0.2 \%$ of females and $0.1 \%$ of males within the age of 15-19 years were infected with HIV and other sexually transmitted infections (STIs). Further, about one third of pregnancies occurring during adolescent age are unintended [16]. Although adolescent discussion with parents regarding sexual and reproductive health issues is crucial in reducing unsafe sexual practices and associated adverse health consequences, literatures in this area are scanty and few researches were also conducted on the magnitude and effect of parent-adolescent discussion about sexual and reproductive health issues. Therefore, this study is motivated by the belief that, barriers in connection with discussions between parents and adolescents can be assessed and described by empirical evidences and investigation and it is, this belief that, the researcher was moved to conduct research in preparatory schools of Hawassa city to fill these knowledge and understanding gaps.

\section{Ethical Clearance}

Ethical clearance was obtained from the CBE Office, College of Medicine and Health Sciences, Hawassa University. The purpose and importance of the study were explained and written consent was obtained from each participant. Confidentiality was maintained throughout the study. The participant involvement was voluntary and those who were non-willing and wanted to quit their participation at any stage were informed to do so without any restriction.

\section{Methodology}

An institutional based descriptive cross sectional research design was conducted among preparatory school students, Hawassa, Ethiopia. The sample size was calculated by using a finite population proportion formula with the following assumptions: $56 \%$ of estimated prevalence, $5 \%$ of marginal error and $95 \%$ of confidence interval (1.96). Since the population size $(\mathrm{N}=3789)$ is less than 10,000 , correction formula was used to calculate the final sample size of 344 . Based on the objectives of the study, the questionnaire was prepared, revised and dispatched to adolescent students. The data were collected by using self-administered questionnaire which contains close ended and open ended questions that comprises on social, economic and cultural factors that hinder the discussion between parents and adolescents. The collected data were checked for its completeness and consistency every day by the researchers. The collected data through questionnaire were edited, coded, entered and cleaned into a computer by using SPSS version 20. Descriptive statistics such as frequencies, percentages, Mean, and cross-tabulation were used to present the study results. Inferential statistics such as binary logistic regression were used to identify the barriers of discussion between parents and adolescents concerning sexual and reproductive health issues in the study area.

\section{Result}

\section{Socio-Demographic Characteristics of Students}

A total of 344 respondents were included in the study with nonon response rate. Out of these respondents, $53.5 \%$ were female students whereas, $45.5 \%$ of them were male students. Age of students was divided into two categories, i.e. between 16-17 and 18-19 years. As data shows, about $22.4 \%$ students were in the range of 16-17 years and $77.6 \%$ were between the age group of 18 19 years. Since age is a continuous variable and measured in years, the average age of students was 17.99. Among the total sample, $75.3 \%$ of students were studying grade 11 , whereas, $24.7 \%$ were attending grade 12 . Regarding respondents of religion, the majority (64.0\%) of them were Protestants, followed by Orthodox (16.7\%). And the rest of the respondents were Catholic (4.9\%), Muslim $(1.7 \%)$ and others were Adventists (2.6\%). Regarding family size of the adolescents, about $50 \%$ of respondents have a family size of 4-6 
family members and $32 \%$ of respondents have 7-9 family members. $10.8 \%$ of them had family size above 9 and the rest $7.3 \%$ of them had less family size which is in between $1-3$.The average family size of adolescent families was 6.5 with a standard deviation of 2.43 .

\section{Social Factors of Adolescents Related to Barriers of} Discussion

A total of 344 participants took part in the study and $73.2 \%$ of their parents live in urban areas with their parents. The majority (78.8\%) of them had no sexual experience but among 73 adolescents those who had sexual experience, about $67.1 \%$ started at the ages of $14-16$ years. About $57.3 \%$ of adolescents follow social media and $52 \%$ of them follow sexual health issues. The majority (89\%) of adolescent parents were married. The majorities $(96.5 \%)$ of adolescents' have knowledge about STD and about 72.6\% used condom in their sexual intercourse. The majority of mothers $(73.3 \%)$ and fathers $(70.7 \%)$ of adolescents were attended formal education.

\section{Economic Factors of Adolescents Related to Barriers of Discussion}

About $47.4 \%$ of adolescents have pocket money and above $50 \%$ of them receiving above 300 birr from their family monthly. More than half $(52.3 \%)$ of the adolescents parents were traders, farmers, daily laborer and NGO employees. The majority (73.6\%) of them were at medium wealth status.

\section{Cultural Factors of Adolescents Related to Barriers of Discussion}

The majority of adolescents did not discuss with their parents (61\%) and their friends (54.9\%) about sex related issues and $72.1 \%$ of them felt shame to talk to family about sex related issues. In the other way, the majority (69.4\%) of adolescents discussed with their brothers, sisters, boyfriends and girlfriends. About $51.5 \%$ of adolescents have watched porn movies and above $64 \%$ of them watched porn movies at the age of 11-16 years. More than half $(59.3 \%)$ of adolescents, have seen sex education books.

\section{Association of Social, Economic and Cultural Barriers of Parent-Adolescent Discussion}

From the survey results it was found that, among adolescents whose family live in urban area, the majority (87.3\%) of them discuss with their family about sex related issues whereas, among adolescents whose family live in rural area the majority $(25.7 \%)$ of them did not discuss with their family about sex related issues. Furthermore, the Chi-square result $\left(x^{2}=8.48, \mathrm{p}<0.01\right)$ shows that, family living place and parent-adolescent discussion status has statistically significant association. Concerning marital status of the adolescents families, the results of Chi-square analysis revealed that, there is no statistically significant association between marital status of adolescent families and parent adolescent discussion status $\left(x^{2}=0.40, p>0.05\right)$. Likewise, Living condition and parentadolescent discussion status has no statistically significant association ( $\left.x^{2}=4.17, \mathrm{p}>0.05\right)$. This indicates that, living condition of adolescents was not a barrier in parent -adolescent discussion about SRH issues. In connection to ever had experienced sexual pleasure, among 271 adolescents those did not experienced sexual pleasure, the majority (88.1\%) of them did not discuss with their parents about sex related issues.

Whereas, among adolescents experienced sexual pleasure the majority (35.8\%) of them discuss with their parents about sex related issues. Furthermore, the Chi-square result $\left(x^{2}=27.99\right.$, $\mathrm{p}<0.01$ ) shows that, ever had experienced sexual pleasure and parent-adolescent discussion status has statistically significant association. On the other hand, age at first pleasure and parentadolescent discussion status has no statistically significant association ( $\left.x^{2}=0.17, \mathrm{p}>0.05\right)$. Respondents were requested the information about whether they used condom or not during their sexual experience, out of the total 53 sampled adolescents those used condom for the first sexual intercourse, the majority (91.7\%) of them discuss with their parents about sex related issues. In contrarily, among 20 adolescents those did not use condom for the first sexual intercourse, the majority $(64.0 \%)$ of them did not discuss with their parents about sex related issues. In addition, the Chi-square result $\left(x^{2}=25.61, \mathrm{p}<0.001\right)$ shows that, using condom and parent-adolescent discussion status has statistically significant association. The adolescents, who follow social media $(70.9 \%)$, discuss with their family more often than those adolescents who did not follow $(29.1 \%)$ and those adolescents who did not follow social media (51.4\%) did not discuss with their family more often than those adolescents who follow social media (48.6\%) about sex related issues.

The results of Chi-square $\left(x^{2}=16.66, \mathrm{p}<0.01\right)$ analysis revealed that, follow up of social media and parent-adolescent discussion status has statistically significant association. Also, following issues on sexual health and parent-adolescent discussion status has no statistically significant association $\left(x^{2}=0.004, p>0.05\right)$. Similarly, the knowledge of adolescents on sexually transmitted diseases and parent-adolescent discussion status has no statistically significant association $\left(x^{2}=1.96, \mathrm{p}>0.05\right)$. This indicates that, the marital status of adolescent families and knowledge of adolescents on sexually transmitted disease was not a barrier of parent adolescent discussion. In connection to educational status of adolescents' parents, whose fathers have completed above grade 12 , the majority (52.2\%) of them discuss with their parents about sex related issues whereas, among adolescents whose fathers did not attend formal education, the majority (43.8\%) of them did not discuss with their parents about sex related issues. Furthermore, the Chi-square result ( $x^{2}=90.97, \mathrm{p}<0.001$ ) shows that adolescents' father educational status and parent-adolescent discussion status has statistically significant association.

In the same way, adolescents' mother educational status and parent-adolescent discussion status has statistically significant association $\left(x^{2}=68.57, \mathrm{p}<0.01\right)$. This indicates that, when adolescents' parents were being educated they have more probability to discuss with their children than parents who has low level of education. The adolescents who have no monthly pocket money (59\%) discuss with their family more often than those adolescents who have monthly pocket money (51.4\%). Also, those adolescents who have monthly pocket money (41\%) discuss with 
their family less often than those adolescents who have no monthly pocket money (48.6\%) about sex related issues. However there was no statistically significant relationship between availability of pocket money and parent-adolescent discussion status $\left(x^{2}\right.$ $=3.54, \mathrm{p}>0.060$ ). Regarding the amount of money adolescents got per month as a pocket money, those adolescents who got above 500birr (46.3\%) did not discusses with their family, whereas, those adolescents who got 100-300 birr (36.4\%) discusses with their family about sex related issues. The Chi-square result $\left(x^{2}=13.02\right.$, $\mathrm{p}<0.01$ ) indicated that the amount of money adolescents' got per a month as pocket money and parent-adolescent discussion status has statistically significant association.

In similar manner, source of adolescents' pocket money $\left(x^{2}\right.$ $=3.25, \mathrm{p}>0.05)$, adolescents' family occupation $\left(x^{2}=3.75, \mathrm{p}>0.05\right)$ and family wealth status $\left(x^{2}=4.34, \mathrm{p}>0.05\right)$ were not found to have statistically significant relationship at $5 \%$ significance level. Talking to friends about sexual related issues was found to be significant. Those adolescents who talk to friends about sexual related issues $(59.5 \%)$ did not discuss with their family more often than those adolescents who talk to friends about sexual related issues (47.8\%). Also, those adolescents who did not talk to friends about sexual related issues $(52.2 \%)$ discuss with their family more often than those adolescents who did not talk to friends about sexual related issues (40.5\%). The Chi-square result $\left(x^{2}=4.57, \mathrm{p}<0.05\right)$ indicates that, a talk to friend about sex related issues and parent-adolescent discussion status has statistically significant association. The

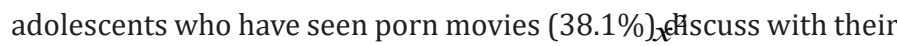
family less often than those adolescents who talk to friends about sexual related issues (40.5\%). Likewise, those adolescents who have not seen porn movies (40\%) discuss with their family more often than those adolescents have not seen porn movies (60\%).

The Chi-square result $\left(x^{2}=15.76, \mathrm{p}<0.01\right)$ indicates that watching porn movies and parent-adolescent discussion status has statistically significant association. In the same way, age at first porn watch and parent-adolescent discussion status has no statistically significant association $\left(x^{2}=0.09, \mathrm{p}>0.05\right)$. Concerning adolescents' experience in reading sex education books, those adolescents who have read sex related books (61.9\%) discuss with their family more often than those adolescents who did not have read sex related Table 1: Binary Logistic Regression Model for the Effects of Independent Variables on Lending Operation Status ( $\mathrm{n}=344$ ).

\begin{tabular}{|c|c|c|c|c|c|}
\hline Variables & B & S.E & Wald & p-Value & Odds Ratio \\
\hline Family living place & 0.062 & .512 & 0.015 & .904 & 1.064 \\
\hline Ever had experienced sexual pleasure & $-1.466^{*}$ & .503 & 8.489 & .004 & 0.231 \\
\hline Follow social media & $1.497^{* *}$ & .643 & 5.423 & .020 & 4.470 \\
\hline Use condom & $2.296^{* *}$ & 1.006 & 5.204 & .023 & 9.934 \\
\hline Father education & $2.398^{* *}$ & .992 & 5.841 & .016 & 10.998 \\
\hline Mother education & $1.582^{* *}$ & .630 & 6.310 & .012 & 4.863 \\
\hline Amount of money & $-2.127^{*}$ & .775 & 7.527 & .006 & 0.119 \\
\hline Talk to friend about sex related issues & $-2.361^{*}$ & .739 & 10.219 & .001 & 0.094 \\
\hline Feel shame to talk to family about sex related issues & $-.868^{* *}$ & .418 & 4.304 & .038 & 0.419 \\
\hline Watch porn & -0.897 & .667 & 1.809 & .179 & 0.408 \\
\hline Constant & -34.936 & 10.592 & 10.878 & .001 & .000 \\
\hline
\end{tabular}

books (42.4\%) did not discuss with their family about sex related issues. But, the relationship between adolescents' experience in reading sex education books and parent-adolescent discussion status were not statistically significant $\left(x^{2}=0.63, \mathrm{p}>0.05\right)$. Similarly, religion of adolescents' parents was not found to have statistically significant relationship with parent-adolescent discussion status $\left(x^{2}=4.49, \mathrm{p}>0.05\right)$. Out of the total sampled respondents those feel shame to talk to family about sex related issues (99\%) did not discuss with their family while those adolescents who did not feel shame (70.1\%) discuss with their family about sex related issues. Additionally, the Chi-square result $\left(x^{2}=194.68, \mathrm{p}<0.001\right)$ indicated that feel shame to talk to family about sex related issues and parentadolescent discussion status has statistically significant association.

\section{Binary Logistic Regression Analysis in Relation to Social, Cultural, and Economic Barriers of Parent Adolescent Discussion}

There were ten variables which were found to be significant in the Chi-square analysis and ready to be tested in binary logistic regression for their overall effect. According to binary logistic regression output, out of ten variables which were included in the model, eight variables such as ever had experienced sexual pleasure, follow social media, use condom, father education, mother education, amount of money, talk to friend about sex related issues and feel shame to talk to family about sex related issues have found to have significant effect on the communication between adolescents and parents. The results of binary logistic regression revealed that, the adolescents who follow social media ( $B=1.497$, $\mathrm{p}<0.05)$, use condom $(\mathrm{B}=2.296, \mathrm{p}<0.05)$, father education $(\mathrm{B}=$ 2.398, $\mathrm{p}<0.05)$ and mother education $(B=1.582, \mathrm{p}<0.05)$ have positive and significant effect on discussion between the parents and adolescents on SRH issues. Culture of adolescents and parents regarding sex related issues whereas, ever had experienced sexual pleasure ( $B=-1.466, p<0.01$ ), amount of money the adolescent got monthly $(B=-2.127, p<0.01)$, talk to friend about sex related issues $(B=-2.361, p<0.01)$, and feel shame to talk to family about sex related issues $(B=-0.868, p<0.05)$ have negative and significant effect on discussion between the adolescents and parents regarding sex related issues. The results were depicted in (Table 1). 


\section{Discussion}

Discussion of sexual and reproductive health issues between parents and adolescents is one of the strategies that could encourage adolescents to delay sexual debut or avoid unprotected sexual intercourse. However, parents and their adolescent children do not often discuss about sexual matters, and even where discussions occur, parents provide scanty information about sexual matters [17]. The objective of this study was to assess the barriers of discussion between the parents and adolescents concerning sexual and reproductive health issues in Preparatory Schools of Hawassa City, SNNPR, Ethiopia. The study revealed that, adolescents discuss with their mothers $(18.7 \%)$ than their fathers $(10.4 \%)$ in their home about SRH issues. Similarly, ever had experienced sexual pleasure, follow social media, use condom at first pleasure, father education status, mother education status, amount of money given to adolescents and talk to friends about sex related issue were found to be the barriers of discussion between parents and adolescents. From the survey results, ever had experienced sexual pleasure and parent-adolescent discussion status has statistically significant association.

Among adolescents those did not experienced sexual pleasure, the majority $(88.1 \%)$ of them did not discuss with their parents about sex related issues whereas, among adolescents experienced sexual pleasure the majority (35.8\%) of them discuss with their parent about sex related issues among adolescents those did not experienced sexual pleasure. Parents expressed the feeling that if they communicate with their children about reproductive health issues they might be directing them to engage in sexual experimentation. They also thought that their children were still too young to know about reproductive health issues [18]. Adolescents who follow social media (70.9\%) discuss with their family more often than those adolescents who did not follow (29.1\%) and those adolescents who did not follow social media (51.4\%) did not discuss with their family more often than those adolescents who follow social media (48.6\%) about sex related issues. Adolescents those had access to television, radio, and internet get the information about reproductive health issue and had high discussion status than those adolescents had no access to social medias [19]. Out of the total sampled adolescents, those used condom for the first sexual intercourse, the majority (91.7\%) of them discuss with their parents about sex related issues.

In contrarily, among adolescents those did not use condom for the first sexual intercourse, the majority (69.6\%) of them did not discuss with their parents about sex related issues. Adolescents who benefited from parental guidance about sex, birth control, and the dangers of STDs were two times more likely to use condoms than adolescents who did not talk to their parents as often. Adolescents, who have repeated communications about sex, sexuality, and development with their parents, are more likely to have an open and closer relationship with them, in addition to being more likely to talk with their parents in the future about sex issues than adolescents whose sexual communication with their parents included less repetition [20]. When adolescents' family was being educated, they have more probability to discuss with their children than families who has low level of education. Among adolescents whose father completed above grade 12 , the majority $(52.2 \%)$ of them discusses with their parent about sex related issues whereas, among adolescents whose father did not attend formal education the majority (43.8\%) of them did not discusses with their parent about sex related issues. In connection to this finding, less educated parents did not discuss about sex education issues with their children. Lack of knowledge about sexual matters inhibited parents from discussing sexual matters with adolescents. Where parents have lack of knowledge about sexual matters hindered communication. They also added that lack of knowledge as a communication barrier makes an individual less confident or skeptical to talk about sexual topics and respond to the concerns raised by children [21]. According to the findings, adolescents who get less money discuss with their family than those adolescents who get more money. Those adolescents who got above 500birr (46.3\%) did not discuss with their family whereas, those adolescents who got 100-300 birr (36.4\%) discuss with their family about sex related issues. World Health Organization reported that, lack of sexuality communication between parents and adolescents exist when adolescents' got excess amount of pocket money because, they always need to enjoy with their friends [1].

Talking to friends about sexual related issues has statistically significant association with parent adolescent discussion. Those adolescents who talk to friends about sexual related issues (59.5\%) did not discuss with their family more often than those adolescents who talk to friends about sexual related issues (47.8\%). In linking to this finding, young people are reluctant to discuss sexuality with their parents since they tend to prefer to discuss these issues with their friends, because they feel shy, and also because they may fear physical punishment for discussing sexuality. The fear of physical punishment or blame was even said to deter reporting to parents that unwanted sex had occurred [18]. As the study result shows, the majority (69.4\%) of adolescents discuss with others (sisters, brothers, boyfriend, girlfriend and etc.), and $18.7 \%$ of them discuss with their mothers, followed by $10.4 \%$ of them discuss with their fathers. Namisi et al, also reported that, in terms of communication, $44 \%$ of adolescents preferred to communicate with mothers about sexuality, while $15 \%$ preferred fathers.

Mothers were the preferred communication partner by the majority of female adolescents in both Tanzania and South Africa. In Cape Town, 31\% preferred discussing with mothers, and $22 \%$ stated a preference for fathers, while in the other two sites, a greater proportion of males preferred discussing with fathers in comparison to mothers about $47 \%$ and $27 \%$ in Dar Es Salaam and Mankweng, respectively [22]. Feel shame to talk to family about sex related issues and parent-adolescent discussion status has statistically significant association. Parents are not expected to discuss with their children issues of physical development and puberty. This task is given to other people who are senior like grandmothers, grandfathers, aunts and uncles. Not only is found to be shameful by parents, society finds it to be an abomination for parents to talk about these issues with their children as the discussion will be about sexuality [23]. 


\section{Conclusion}

The majority of respondents (61\%) did not discuss sexual matters with their parents. Some parents believed discussions on sexual matters with adolescents as a means that could entice them to indulge in sexual intercourse. Other parents also believe adolescents as too young to discuss sexual matters while others understand adolescents as knowledgeable since they learn from the peers and media. Therefore, the adolescents continue to have lacking information from parents. In order to solve this problem, meetings are interpersonal channels of discussion and if they are regularly scheduled, discussion between parents and adolescent could be improved. Therefore, parent-adolescent discussion is a necessary issue. The major factors that hindered parent-adolescent discussion were, ever had experienced sexual pleasure, follow social media, use condom, parent education, amount of money given to adolescents, talk to friends about sex related issue, and feel shame. Furthermore, parents had limited access to sexuality information which could perpetuate beliefs, taboos and negative attitudes that hindered them from discussing sexual matters with adolescents.

\section{Acknowledgement}

We would like to thank Hawassa University, College of Medicine and Health Science, CBE office, School of Nursing and Midwifery for giving Support throughout the study. We would like to express our heartfelt gratitude to our students who filled the questionnaire and school teachers for their contribution during data collection. We would also like to forward our deepest thank and respect for those persons and offices of Hawassa city administration education department who gave us important base line information.

\section{References}

1. (2011) World Health Organization, Unsafe abortion global and regional estimates of the incidence of unsafe abortion and associated mortality in 2008.

2. (2006) Federal Democratic Republic of Ethiopia Ministry of Health (FDREMoH). National adolescent and youth reproductive health strategy 2006-2015.

3. Jewkes R (2010) Where to for sexual health education for adolescents in Sub-Saharan Africa? PLoS Medicine 8(6): 1000288.

4. Mutema F (2013) Breaking the Silence: Discussion between parents and secondary school adolescents in the context of HIV/AIDs in Zimbabwe: A Case of Mkoba High Density Suburb. Gweru Journal of Emerging Trends in Educational Research and Policy Studies 4(4): 604-612.

5. Bastien S, Kajula L, Muhwezi W (2011) A review of studies of parentchild discussion about sexuality and HIV/AIDS in Sub-Saharan Africa. Reproductive health 8(25).

6. Adu-Mireku S (2003) Family discussion about HIV/AIDS and sexual behavior among senior secondary school students in Accra Ghana. Africa Health Science 3: 7-14.
7. Berg K, Sun CJ, Babalola S (2012) Predictors of parent-child discussion among a nationally representative sample in Nigeria. AHARA-J. Journal of Social Aspects of HIV/AIDS 9(2): 95-103.

8. Wamoyi J, Fenwick A, Urassa M, Zaba B, Stones W (2010) Parent-child discussion about sexual and reproductive health in rural Tanzania: Implications for the young people's sexual health interventions reproductive health $7(6)$ : 4755 .

9. (2005) Ministry of Youth, Sport and Culture of Ethiopia (MYSC), National Sport Policy.

10. Eisenberg LH, Sieving RE, Bearinger LH, Swain C, Resnick MD (2006) Parents' Discussion with Adolescents about Sexual Behavior: A missed Opportunity for Prevention. Journal of Youth Adolescence 35(6): 893902.

11. Ahlberg BM, Jylk"as E, Krantz I (2001) Gendered construction of sexual risks: implications for safer sex among young people in Kenya and Sweden. Reproductive Health Matters 9(17): 26-36.

12. Berhanu L, Gail D (2005) Young peoples' HIV/AIDS and reproductive health needs and utilization of services in selected region of Ethiopia. Ethiopian Public Health Association 79.

13. Huberman B (2002) Raising sexually healthy youth: right, respect, responsibility \& parent-child discussion. Transitions 15(1): 1-4.

14. Wang B, Li X, Stanton B, Kamali V, Naar-King S, et al. (2007) Sexual attitudes, pattern of discussion, and sexual behavior among unmarried out-of school youth in China. BMC Public Health 7: 189.

15. UNAIDS (2010) UNAIDS report on the Global AIDS Epidemic. Geneva: Joint United Nations Program on HIV/AIDS (UNAIDS).

16. Tebekaw Y, Aemro B, Teller C (2014) Prevalence and determinants of unintended childbirth in Ethiopia. BMC pregnancy and childbirth 14: 326.

17. Ogle S, Glasier A, Riley SC (2008) Communication between parents and their children about sexual health. Contraception 77(4): 283-288.

18. Kumi-Kyereme A, Awusabo-Asare K, Biddlecom A, Tanle A (2007) Influence of social connectedness, communication and monitoring on adolescent sexual activity in Ghana. African Journal Reproductive Health 11(3): 133-149.

19. Odek T (2006) Cultural Challenges and Sex Education in Mageta Islands. Post-Sexuality Leadership Development Fellowship Report Series Number 3.

20. Weinman M, Small E, Buzi RS, Smith P (2008) Risk Factors, Parental Communication, Self and Peers' Beliefs as Predictors of Condom Use Among Female Adolescents Attending Family Planning Clinics. Child Adolescent Social Work Journal 25:157-170.

21. Guilamo-Ramos V, Bouris A (2008) Parent-Adolescent Communication about Sex in Latino Families. A Guide for Practitioners.

22. Namisi FS, Flisher AJ, Overland S, Bastien S, Onya H, et al. (2009) Sociodemographic variations in communication on sexuality and HIV/ AIDS with parents, family members and teachers among in-school adolescents: a multi-site study in Tanzania and South Africa. Scandinavia Journal Public Health 37(2): 65-74.

23. Mbugua N (2007) Factors inhibiting educated mothers in Kenya from giving meaningful sex-education to their daughters. Social Science Medical journal 64 (5): 1079-1089. 


A Assets of Publishing with us
BESEDICAL

